\title{
Comparison of the Effects of Myristoylated and Transactivating Peptide (TAT) Conjugated Mitochondrial Fission Peptide Inhibitor (P110) in Myocardial Ischemia/Reperfusion (I/R) Injury
}

\author{
Israel Benjamin, Jonathan Vu, Christina Lipscombe, Devon Stutzman, \\ Carly Schmidgall, Harsh Patel, Samir Patel, Qian Chen, Cathy J. Hatcher, \\ Robert Barsotti, and Lindon H. Young \\ Division of Research, Department of Bio-Medical Sciences, Philadelphia College of Osteopathic Medicine
} (PCOM), Philadelphia, PA, 19131, USA

\section{Introduction}

Mitochondrial dynamics, mitochondrial fusion and fission may be involved in myocardial ischemia/reperfusion (MI/R) injury. In particular, mitochondrial fission is associated with mitochondrial fragmentation and decreased ATP production leading to cardiac contractile dysfunction and increased infarct size in MI/R [1-3]. During ischemic events, coronary blood flow is restricted causing cardiomyocytes to enter a hypoxic state. This change in cellular respiration causes a buildup of lactic acid and a decrease in $\mathrm{pH}$. The acidic conditions developed during ischemia prevent the opening of the mitochondrial permeability transition pore (MPTP) and cause cardiomyocyte hypercontracture. When blood flow and oxygen delivery are restored during reperfusion, reactive oxygen species (ROS) are generated which leads to the loss of mitochondrial membrane potential and opening of the MPTP, which potentiates mitochondrial fission in MI/R (Figure 1). Therefore inhibiting mitochondrial fission, which results from the vital act of reperfusion, may be a strategy to salvage damaged cardiomyocytes and protect them from MI/R injury. P110 (DLLPRGT) is a mitochondrial fission peptide inhibitor that acts by selectively inhibiting the interaction between human fission protein (Fis1), which is located on the outer mitochondrial membrane and dynamin related protein 1 (Drp1), a GTPase (Figure 2).

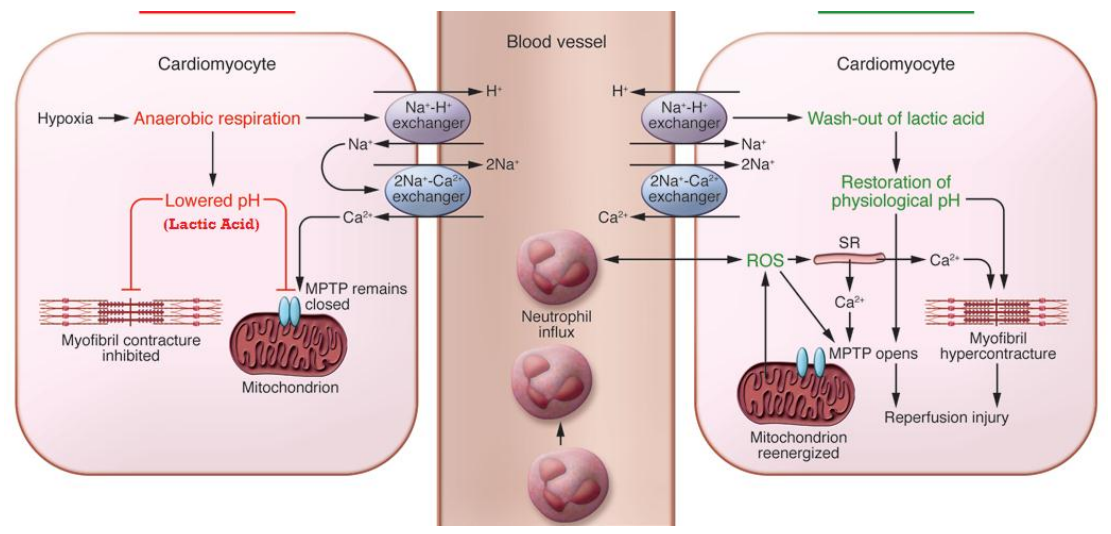

Fig. 1. Acute myocardial ischemia results in a decrease in pH from anaerobic conditions. The acidic conditions during ischemia prevent the opening of the mitochondrial permeability transition pore $(M P T P)$ and cardiomyocyte hypercontracture at this time. Reperfusion results in rapid restoration of physiological $\mathrm{pH}$, which promotes opening of the MPTP, reactive oxygen species (ROS) release, $\mathrm{Ca}^{2+}$ overload, and cardiomyocyte hypercontracture. Neutrophils accumulate in the infarcted myocardial tissue in response to the release of chemoattractants, and generate ROS leading to further tissue damage. Adapted from Hausenloy and Yellon, 2013 [1]. 


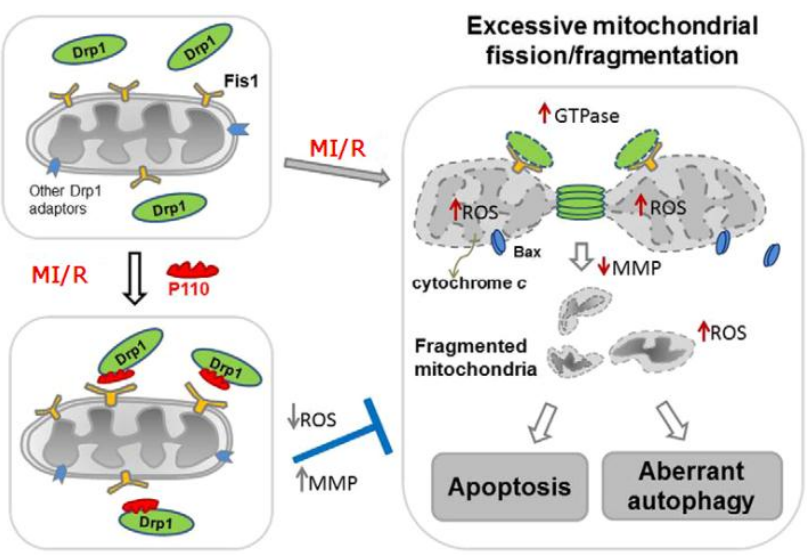

Fig. 2. Mechanism of mitochondrial fission in myocardial ischemia/reperfusion (MI/R) and P110's inhibition of interaction between dynamin related protein 1 (Drpl) and human fission protein (Fisl). Mitochondrial membrane potential (MMP) is increased and reactive oxygen species (ROS) are decreased with P110. Adapted from Qi, 2013 [3].

It is well known that adding myristic acid (Myr) or TAT carrier peptide to native peptides will facilitate cell membrane permeability required for targeting intracellular substrates. The addition of a glycineglycine spacer between the TAT and cargo portion of the peptide is reported to facilitate delivery of the cargo sequence (i.e., DLLPRGT) [3,4]. Myristic acid conjugated peptides enter into cells by simple diffusion whereas TAT-conjugated peptides enter into cells via an endocytosis-like mechanism involving interactions with positively-charged amino acids (i.e., RKKRRQRRR) and negatively charged plasma cell membrane [4,5]. Native peptides enter through facilitated diffusion (Figure 3). We have previously shown that myristic acid conjugated caveolin-1 and PKC $\beta I I$ and $\zeta$ peptide inhibitors significantly attenuated leukocyte chemotactic receptor stimulated superoxide release compared to their native counterparts [5]. However, it is not known if differences exist in the effectiveness of myristic acid versus TAT-conjugated peptides compared to their native counterparts or untreated controls in MI/R. In the current study, we compared the effects of TAT-conjugated P110 $(\mathrm{MW}=2427 \mathrm{~g} / \mathrm{mol}), \mathrm{Myr}-\mathrm{P} 110(\mathrm{MW}=981 \mathrm{~g} / \mathrm{mol})$, and Native-P110 (MW=771 g/mol) on cardiac function and infarct size in isolated perfused rat hearts. Previously it was shown that TAT-P110 exerted a modest (i.e. one-third) reduction in infarct size compared to untreated controls; however, cardiac function was not reported in the study, nor was there a comparison of TAT-P110 to native peptide in isolated perfused rat hearts subjected to $\mathrm{MI}(30 \mathrm{~min}) / \mathrm{R}(90 \mathrm{~min})$ [2].

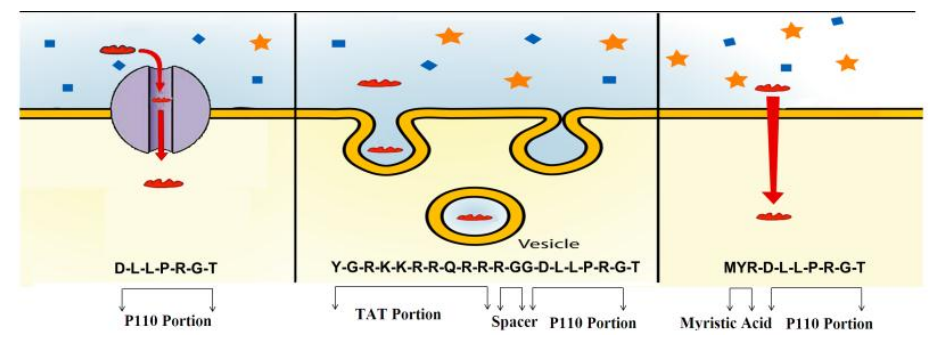

Fig. 3. Cell entry mechanisms for Native, TAT, and MyrP110. The a.a. sequence of TAT, GG spacer, and P110 is depicted above.

\section{Results and Discussion}

Male Sprague Dawley rats (275-325 g, Charles River, Springfield, MA) were anesthetized with pentobarbital sodium $(60 \mathrm{mg} / \mathrm{kg})$ and anti-coagulated with sodium heparin $(1,000 \mathrm{U})$ injection intraperitoneally. Each heart was rapidly excised, immersed in a $160 \mathrm{~mL}$ water-jacketed reservoir and subjected to retrograde perfusion via the aorta with a modified Krebs-Henseleit buffer. The perfusate was maintained at $37^{\circ} \mathrm{C}$, kept at $80 \mathrm{mmHg}$ constant pressure, aerated with $95 \% \mathrm{O}_{2}-5 \% \mathrm{CO}_{2}$, and equilibrated at a pH of 7.35-7.45. A flow meter (T106, Transonic Systems, Inc., Ithaca, NY) placed in the inflow line monitored coronary flow. Left ventricular end-systolic pressure (LVESP), left ventricular end-diastolic pressure (LVEDP), heart rate, and peak rates of rise and fall in the first derivative $\left(\mathrm{dP} / \mathrm{dt}_{\max }\right.$ and $\mathrm{dP} / \mathrm{dt}_{\min }$, respectively) of left ventricular developed pressure (LVDP) were monitored using a pressure transducer (SPR-524, Millar Instruments, Inc., Houston, TX) positioned in the left ventricular cavity and recorded using a Powerlab Station acquisition system (ADInstruments, 
Grand Junction, CO). LVDP was calculated by subtracting LVEDP from LVESP. Sham hearts were not subjected to global ischemia. At the end of reperfusion, five heart slices $(2 \mathrm{~mm} / \mathrm{slice})$ were subjected to $1 \%$ triphenyltetrazolium chloride (TTC) staining to detect non-viable (unstained) and viable (red stained) area. Infarct size was expressed as the percentage of non-viable tissue to the total tissue weight [6]. All data are presented as means \pm SEM. Cardiac function and TTC staining data were analyzed by ANOVA using post hoc analysis with the Student-Newman-Keuls test. Probability values of $<0.05$ were considered statistically significant.

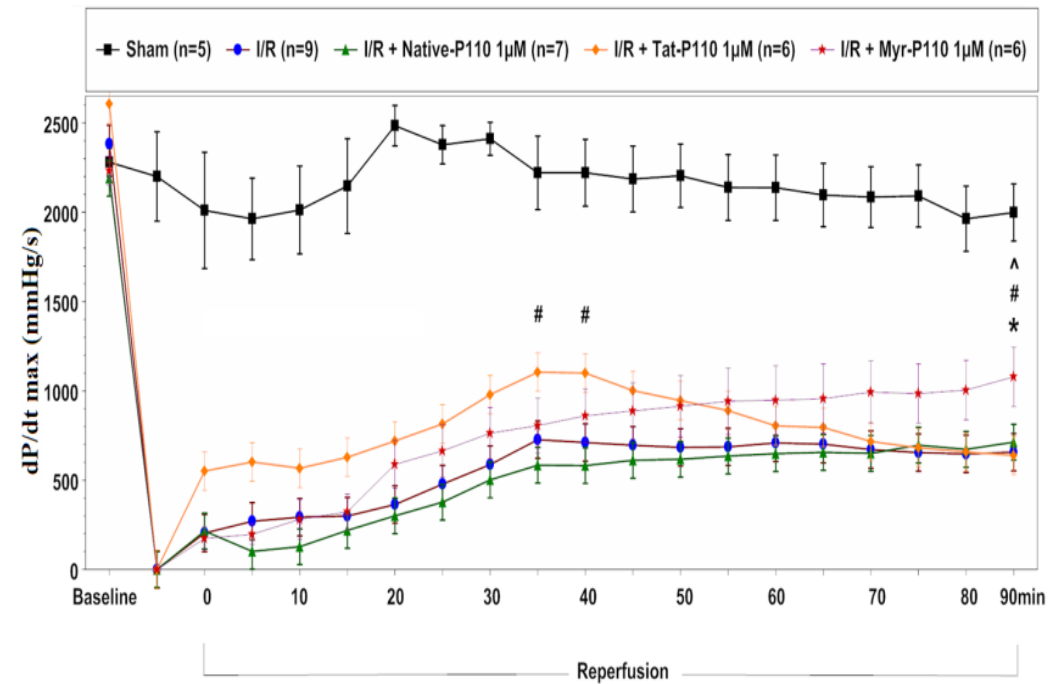

Fig. 4. Time course of $d P / d t_{\max }$ for Sham, MI/R and MI/R + Tat-P110, Myr-P110 and Native P110 hearts. $\left(* p<0.05\right.$ compared to untreated I/R hearts; \# $p<0.05$ compared to Native hearts; ${ }^{\wedge} p<0.05$ compared to TAT-P110 hearts).

Figure 4 shows that Myr-P110 significantly improved post-reperfused dP/dtmax compared to untreated MI/R, TAT-P110, and Native-P110 hearts. Sham hearts maintained cardiac function throughout the experimental protocol $(87 \pm 9 \%$ of initial LVDP (data not shown) and $89 \pm 8 \%$ of initial dP/dtmax. We found that Myr-P110 ( $1 \mu \mathrm{M} ; \mathrm{n}=6)$ given for $10 \mathrm{~min}$ before ischemia and for $20 \mathrm{~min}$ post-reperfusion, significantly restored $\mathrm{dP} / \mathrm{dtmax}$ to $49 \pm 7 \%$ compared to TAT-conjugated P110 (1 $\mu \mathrm{M} \mathrm{n}=6$ ), Native P110, and untreated controls ( $n=9)$, which only recovered to $26 \pm 5 \%, 35 \pm 6 \%, 28 \pm 4 \%$ of baseline values at $90 \mathrm{~min}$ post-reperfusion respectively. TAT-P110 transiently improved post-reperfused $\mathrm{dP} / \mathrm{dtmax}$ at 40-45 minutes compared to untreated controls, but this effect diminished by the end of reperfusion.

Weight ratios of infarcted to area at risk left ventricular tissue in MI/R were determined by TTC staining. Myr-P110 significantly reduced infarct size to $28 \pm 2 \%$ compared to untreated MI/R controls which had an infarct size of $46 \pm 3 \%(\mathrm{p}<0.01)$. Whereas, TAT-conjugated P110 and Native P110 treated hearts had an infarct size of $35 \pm 3 \%$ and $34 \pm 3 \%$ respectively, and was not statistically different from controls using ANOVA analysis. Coronary flow ( $\mathrm{ml} / \mathrm{min}$ ) (data not shown) was not statistically different among the experimental groups during initial baseline or reperfusion. It is known that coronary flow in the ex vivo isolated perfused rat heart model can have a wide variation of postreperfusion coronary flow (i.e. 7 to $14 \mathrm{ml} / \mathrm{min}$ ) and this variable is independent of post-reperfused cardiac function within the $7-14 \mathrm{ml} / \mathrm{min}$ range among all MI/R study groups [7].

In summary, these data suggest that mitochondrial fission may contribute to MI/R injury and that inhibition of mitochondrial fission by Myr-P110 significantly improved post-reperfused dP/dt $\max _{\text {ax }}$ and reduced infarct size as compared to untreated control, TAT, and Native P110 MI/R hearts. Interestingly, TAT-P110 transiently improved post-reperfused cardiac function but did not significantly reduce infarct size compared to Native P110. The data suggest that TAT-P110 had limited intracellular entry compared to Myr-P110, and therefore only transiently improved cardiac function and was marginally effective in reducing infarct size. Future studies will be aimed at attaching a 


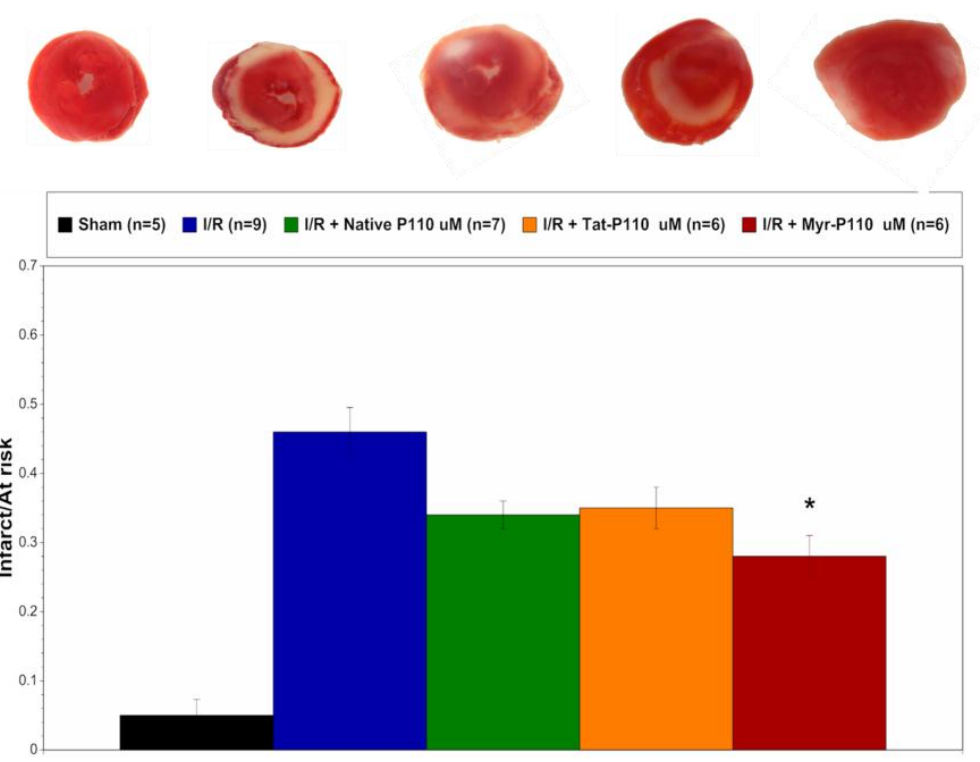

Fig. 5. Representative 1\% triphenyltetrazolium chloride (TTC) stained heart sections displayed above from sham, control MI/R and MI/R + P110 treated hearts were used to assess infarct size after cardiac function experiments (*p<0.05 compared to untreated MI/R hearts.) Myr-P110 $1 \mu M$ significantly reduced infarct size compared to untreated MI/R hearts. Sham hearts had minimal cell death $(<5 \%)$ at the end of the experimental protocol.

fluorescent probe to both TAT and Myr-P110 peptides to monitor intracellular targets and the time course of cell entry and degradation of both peptide formulations. Moreover, additional studies using higher concentrations, i.e. $5 \mu \mathrm{M}$ of both TAT and Myr-P110 will be tested in our MI/R model. The data suggests that Myr-P110 is the more effective formulation and may be an attractive strategy to attenuate $\mathrm{MI} / \mathrm{R}$ injury and salvage heart tissue in MI patients.

\section{Acknowledgments}

This study was supported by the Center for Chronic Disorders of Aging and the Department of Bio-Medical Sciences at PCOM. Special thanks to PCOM DO/Biomed students Stephanie Liu, Joseph Venditto, and Gregory Stoner for assistance in the preparation of this manuscript.

\section{References}

1. Hausenloy, D.J., Yellon, D.M. J. Clin. Invest. 123, 92-100 (2013), http://dx.doi.org/10.1172/JCI62874

2. Disatnik, M.H., et al. J. Am. Heart Assoc. 2, e000461 (2013), http:///dx.doi.org/10.1161/JAHA.113.000461

3. Qi, X., et al. J. Cell Sci. 126, 789-802 (2013), Advance Online Article December 13, 2012, http://dx.doi.org/10.1242/jes.114439

4. Taraballi, F., et al. Frontiers in Neuroengineering 3, (2010), http://dx.doi.org/10.3389/neuro.16.001.2010

5. Perkins, K.A., et al. in Lebl, M. (Ed.) Building Bridges (Proceedings of the 22nd American Peptide Symposium), American Peptide Society, San Diego, 2011, p. 288-289.

6. Blakeman, N., et al. Am. J. Biomed. Sci. 4, 249-261 (2012), http://dx.doi.org/10.5099/aj120300252

7. Peterman, E.E., Taormina, II P., Harvey, M., Young, L.H. J. Cardiovasc. Pharmacol. 43, 645-656 (2004), http://dx.doi.org/10.1097/00005344-200405000-00006 